\title{
BMJ Open Study protocol for a systematic review and meta-analysis of comorbidities and stroke characteristics associated with troponin elevation after acute stroke
}

\author{
Amado Jimenez-Ruiz (DD , ${ }^{1}$ Sebastian Fridman (D) , ${ }^{2}$ Luciano A Sposato (1) ${ }^{1,2,3,4,5,6}$
}

To cite: Jimenez-Ruiz A, Fridman S, Sposato LA. Study protocol for a systematic review and meta-analysis of comorbidities and stroke characteristics associated with troponin elevation after acute stroke. BMJ Open 2021;11:e043613. doi:10.1136/ bmjopen-2020-043613

- Prepublication history and additional material for this paper is available online. To view these files, please visit the journal online (http://dx.doi.org/10. 1136/bmjopen-2020-043613).

Received 10 August 2020 Revised 08 December 2020 Accepted 11 January 2021

Check for updates

(C) Author(s) (or their employer(s)) 2021. Re-use permitted under CC BY-NC. No commercial re-use. See rights and permissions. Published by BMJ.

For numbered affiliations see end of article.

Correspondence to

Dr Luciano A Sposato;

lucianosposato@gmail.com

\section{ABSTRACT}

Introduction It is unknown which comorbidities and stroke characteristics are associated with elevated cardiac troponin (cTn) levels after stroke. The main objective of this systematic review and meta-analysis is to assess the association of elevated cTn with preexisting cardiovascular comorbidities (eg, coronary artery disease, heart failure and structural heart disease), specific stroke characteristics (eg, infarct/haemorrhage size, stroke severity, insular cortex involvement) and renal failure after ischaemic stroke (IS) or intracranial haemorrhage (ICH). The secondary objective is to evaluate the association of elevated cTn with stroke recurrence and death.

Methods and analysis We will include all crosssectional, case-control, cohort studies and clinical trials involving IS and ICH adult patients ( $\geq 18$ years), published between 1 January 1990 and 31 December 2020 in English or Spanish, reporting the proportion with elevated cTn. We will search PubMed, EMBASE and Web of Science by applying predefined search terms. Two reviewers will independently screen titles and abstracts, retrieve full texts, extract the data in a predesigned form, and assess the risk of bias. We will apply random-effects or fixedeffects meta-analyses to estimate the association between cardiovascular comorbidities, stroke characteristics and renal failure with cTn elevation. We will report results as risk ratios or $\mathrm{ORs}$. We will perform sensitivity analyses for subtypes of cTn (cTn-I and cTn-T), regular versus highsensitivity assays, and type of stroke (IS vs ICH). We will estimate heterogeneity by using $\mathrm{t}^{2} Q$ and $\mathrm{I}^{2}$ measures. We will use funnel plots, Rosenthal's Fail-Safe N, Duval and Tweedie's trim and fill procedure, and Egger's regression intercept to assess publication bias.

Ethics and dissemination This review will be based on published data and does therefore not require ethical clearance. The results will be published in peer-reviewed journals.

PROSPERO registration number CRD42020203126.

\section{INTRODUCTION}

As part of current best practice recommendations, cardiac troponin (cTn) is routinely measured in patients with acute stroke. ${ }^{1}$ It has been suggested that cTn elevation after stroke is caused by acute myocardial injury triggered by neurogenic mechanisms in patients with or
Strengths and limitations of this study

- This study will help to identify markers associated with cardiac troponin (cTn) elevation among patients with ischaemic and haemorrhagic stroke, and whether cTn elevation is associated with stroke recurrence and death in this population.

- We will apply well-validated systematic review and meta-analysis tools that are fully compliant with current international guidelines and recommendations.

- As a limitation, we expect to find large heterogeneity between study populations, study designs and types of exposure; we will, thus, attempt to apply metaregression techniques and subgroup analyses top account for these limitations.

- Another limitation of this study is that despite describing variables associated with troponin cTn elevation, we may not be able to identify the mechanisms underlying those associations.

- Despite of the latter limitation, we expect, at least, to provide a mechanistic hypothesis for this association, which will still represent an important contribution to the better understanding the pathophysiology of myocardial injury in patients who had a stroke.

without underlying heart disease. ${ }^{23}$ Current understanding of these neurogenic mechanisms indicates that they comprise autonomic dysfunction and an excessive inflammatory response leading to structural and functional changes in the myocardium even in the absence of coronary artery disease or myocardial ischemia (eg, through non-ischaemic mechanisms). ${ }^{4}$ An alternative or complementary explanation is that elevated levels of cTn found among patients who had a stroke are the consequence chronic myocardial injury associated with prevalent risk factors (eg, hypertension), renal failure or the expression of underlying heart disease. Essential cardiac comorbidities associated with chronically elevated cTn include coronary artery disease, atrial fibrillation, congestive heart failure and left ventricular hypertrophy. Importantly, 
subclinical chronic myocardial injury (elevated cTn in individuals without clinically evident heart disease or stroke) is associated with increased long-term risk of stroke. $^{5}$

Which specific cardiovascular comorbidities or stroke characteristics are associated with increased cTn levels after stroke, remains unknown. This is a relevant question that needs to be answered to better understand the pathophysiology, risk and outcomes of elevated cTn levels among acute stroke patients. Clinically, an acute poststroke rise and fall of cTn levels $>20 \%$ has been proposed as a surrogate of acute post-stroke myocardial injury. ${ }^{6}$ While this is the ideal approach for identifying patients among whom neurogenic mechanisms play a role, studies reporting rise and fall patterns are scarce. From a theoretical and mechanistic approach, and as a way of overcoming this limitation, we hypothesised that if transient cTn elevation is the consequence of neurogenic mechanisms, factors associated with the severity of the stroke (eg, stroke severity or infarct/haemorrhage size) or the involvement of cerebral structures that regulate cardiac autonomic function (eg, insular involvement) would show an association with acute post-stroke cTn elevation in studies in which serial measurements of cTn levels are not available. For studies in which serial measurements of cTn are reported, we hypothesise that cTn rise and fall patterns will be associated with stroke severity, the size of brain ischaemic or haemorrhagic brain lesions or the involvement of the insular cortex. To address these knowledge gaps, we will conduct a systematic review and meta-analysis of studies including ischaemic stroke (IS) and intracranial haemorrhage (ICH) patients reporting cTn levels-or cTn rise and fall patterns when available, to estimate the association of increased cTn with specific cardiovascular comorbidities and stroke characteristics. We will also assess the risk of stroke recurrence and death among IS and ICH patients with elevated cTn.

\section{REVIEW QUESTIONS}

- Is there an association between elevated cTn levels post-stroke and specific cardiovascular comorbidities or stroke characteristics?

- Is elevated cTn associated with increased risk of stroke recurrence or death?

\section{OBJECTIVES}

- Primary objective: To estimate the association of elevated cTn levels with vascular risk factors, cardiovascular comorbidities (eg, hypertension, diabetes mellitus, dyslipidaemia, coronary artery disease, heart failure, atrial fibrillation and structural heart disease defined as either left atrial enlargement or decreased left ventricular ejection fraction) and stroke characteristics (eg, stroke severity, cerebral infarct/haemorrhage size and insular involvement).

- Secondary objectives: To estimate the association of elevated cTn levels with the risk of stroke recurrence, death and stroke recurrence or death. To estimate the proportion of patients showing predefined electrocardiographic changes (ST-T changes, QT prolongation or atrial fibrillation detected after stroke).

\section{METHODS}

This study protocol has been prepared according to the 2015 Preferred Reporting Items for Systematic Reviews and Meta-Analyses Protocols guidelines (online supplementary appendix $)^{7}$.

\section{Criteria for considering studies for the review Inclusion criteria}

We will include all cross-sectional, case-control and cohort studies and clinical trials published between 1 January 1990 and 31 December 2020 in English or Spanish involving adults (18 years of age or older) and reporting on the prevalence of elevated cTn after stroke:

- IS or ICH (excluding isolated subarachnoid haemorrhage).

- Studies reporting serum/plasma cTn of any type and assay, measured within 7 days of the event.

- Available data on the proportion with high cTn.

- Prospective or retrospective cohort studies.

\section{Exclusion criteria}

We will exclude reviews, letters to the editor, editorials, conference articles with incomplete data, studies with a small sample size (less than 30 participants). For duplicated publications (reports including the same population), we will collate multiple reports to craft the most comprehensive database from that study.

\section{Search strategy for the identification of relevant studies}

We will search PubMed, EMBASE and Web of Science to identify potentially eligible studies by applying predefined search terms. Search terms are shown in tables 1-3. We will also use the 'similar articles' PubMed function (first 50 articles listed per article included in the study), we will screen the reference lists of included articles and we will search each of this study authors' personal archives for additional relevant publications that were not identified in the study search.

\section{Selection of studies for inclusion in the review}

Two reviewers will independently screen titles and abstracts by using COVIDENCE and will solve disagreements by consensus. In cases of persisting disagreement, a third reviewer will intervene. The same reviewers will fully assess all potentially relevant records. We will document reasons for excluding specific publications.

\section{Assessment of the methodological quality and risk of bias}

To evaluate the methodological quality and risk of bias of each publication, we will use the risk of bias in nonrandomised studies of interventions (ROBINS-I) ${ }^{8}$ on six domains: bias due to confounding, bias in selection of participants, bias in classification of interventions, bias due to deviations from intended interventions, bias due 
Table 1 PubMed search terms

\begin{tabular}{|c|c|}
\hline Searches & Results \\
\hline 1 & 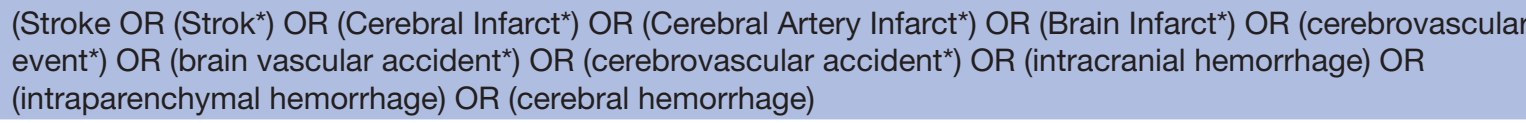 \\
\hline 2 & Troponin [Mesh] OR Troponin [tiab] OR myocardial injury [Tiab] \\
\hline 3 & Animals NOT Humans \\
\hline 4 & NOT case reports OR case report NOT Clinical Study OR series \\
\hline 6 & \#5 NOT \#3 \\
\hline 7 & \#6 NOT \#4 \\
\hline 8 & \#7 Timespan 1990-December $31^{\text {st }} 2020$ \\
\hline
\end{tabular}

to missing data, bias in measurement of outcomes, and bias in selection of the reported result. We will classify the results following the ROBINS-I criteria as low, moderate, serious, critical risk of bias, or no information. We will present a risk-of-bias graph and summary.

\section{Data extraction and management}

- We will create and use a standardised COVIDENCE data extraction form including the following.

- Study identification: funding source, country, setting, author name, institution, email, address and possible conflicts of interest.

- Study characteristics: study design, groups, aim of the study, start date, inclusion and exclusion criteria, recruitment methods and setting.

- Patients' characteristics: mean or median age, stroke severity as determined by the mean or median National Institutes of Health stroke scale, mean or median interval time to cTn measurement,

\begin{tabular}{|c|c|}
\hline Searches & Results \\
\hline 1 & $\begin{array}{l}\text { cerebrovascular accident/ or cardioembolic } \\
\text { stroke/ or lacunar stroke/ }\end{array}$ \\
\hline 2 & $\begin{array}{l}\text { (strok }^{*} \text { or }\left(\text { cerebral adj2 infarct }{ }^{\star}\right) \text { or (brain adj2 } \\
\left.\text { infarct }^{\star} \text { ) or cerebrovascular event }{ }^{\star}\right) . t i, \text { ab. }\end{array}$ \\
\hline 3 & exp troponin/ \\
\hline 4 & troponin.ti,ab. \\
\hline 5 & 1 or 2 \\
\hline 6 & 3 or 4 \\
\hline 7 & 5 and 6 \\
\hline 8 & 7 \\
\hline 9 & limit 8 to human \\
\hline 10 & $\begin{array}{l}\text { (case report* or systematic review or meta } \\
\text { analysis or meta-analysis).ti,ab. }\end{array}$ \\
\hline 11 & (series or clinical study).ti,ab. \\
\hline 12 & 10 not 11 \\
\hline 13 & 9 not 12 \\
\hline 14 & limit 13 to $d d=19900101-20201231$ \\
\hline
\end{tabular}

hypertension $(\mathrm{n})$, diabetes mellitus $(\mathrm{n})$, chronic kidney disease (n), dyslipidaemia (n), active smoking $(n)$, alcohol misuse $(n)$, coronary artery disease $(n)$, prior myocardial infarction (n), heart failure (n), atrial fibrillation $(\mathrm{n})$, prior IS $(\mathrm{n})$, prior transient ischaemic attack (\%), prior ICH (n), dementia (n), Trial of ORG 10172 in Acute Stroke Treatment category (n), embolic stroke of undetermined source (n), insular involvement $(\mathrm{n})$, brain infarct/haemorrhage volume $(\mathrm{mL})$, impaired left ventricular ejection fraction $(n)$, impaired left ventricular ejection fraction $(n)$, left ventricular ejection fraction $(\%)$, enlarged left atrium (n), left atrial size (diameter, area, volume or volume index depending on data availability), mortality (\%, HR, OR, etc), recurrent stroke (\%, HR, OR, etc), ST-changes (n), QT prolongation (n) and atrial fibrillation detected after stroke (n).

- Main exposure: proportion of patients with rise and fall cTn pattern when available. When unavailable, we will use elevated cTn. Exposure characteristics:

\begin{tabular}{|c|c|}
\hline Searches & Results \\
\hline 1 & $\begin{array}{l}\text { TS }=(\text { stroke OR (brain NEAR infarct*) OR (cerebral } \\
\text { NEAR infarct*) OR cerebrovascular accident* OR } \\
\left.\text { cerebrovascular event }^{\star}\right)\end{array}$ \\
\hline 2 & TS=(troponin* OR (myocardial NEAR injury*)) \\
\hline 3 & $\begin{array}{l}\mathrm{Tl}=(\text { ("case report" NOT ("clinical study" OR } \\
\text { "series")) }\end{array}$ \\
\hline 4 & $\begin{array}{l}\text { Tl=(veterinary OR rabbit OR rabbits OR animal } \\
\text { OR animals OR mouse OR mice OR rodent OR } \\
\text { rodents OR rat OR rats OR pig OR pigs OR } \\
\text { porcine OR horse* OR equine OR cow OR cows } \\
\text { OR bovine OR goat OR goats OR sheep OR ovine } \\
\text { OR canine OR dog OR dogs OR feline OR cat OR } \\
\text { cats) }\end{array}$ \\
\hline 5 & \#1 AND \#2 \\
\hline 6 & \#5 NOT \#3 \\
\hline 7 & \#6 NOT \#4 \\
\hline 8 & \#7 Timespan 1990-31 December 2020 \\
\hline
\end{tabular}


Table 4 Study outcomes

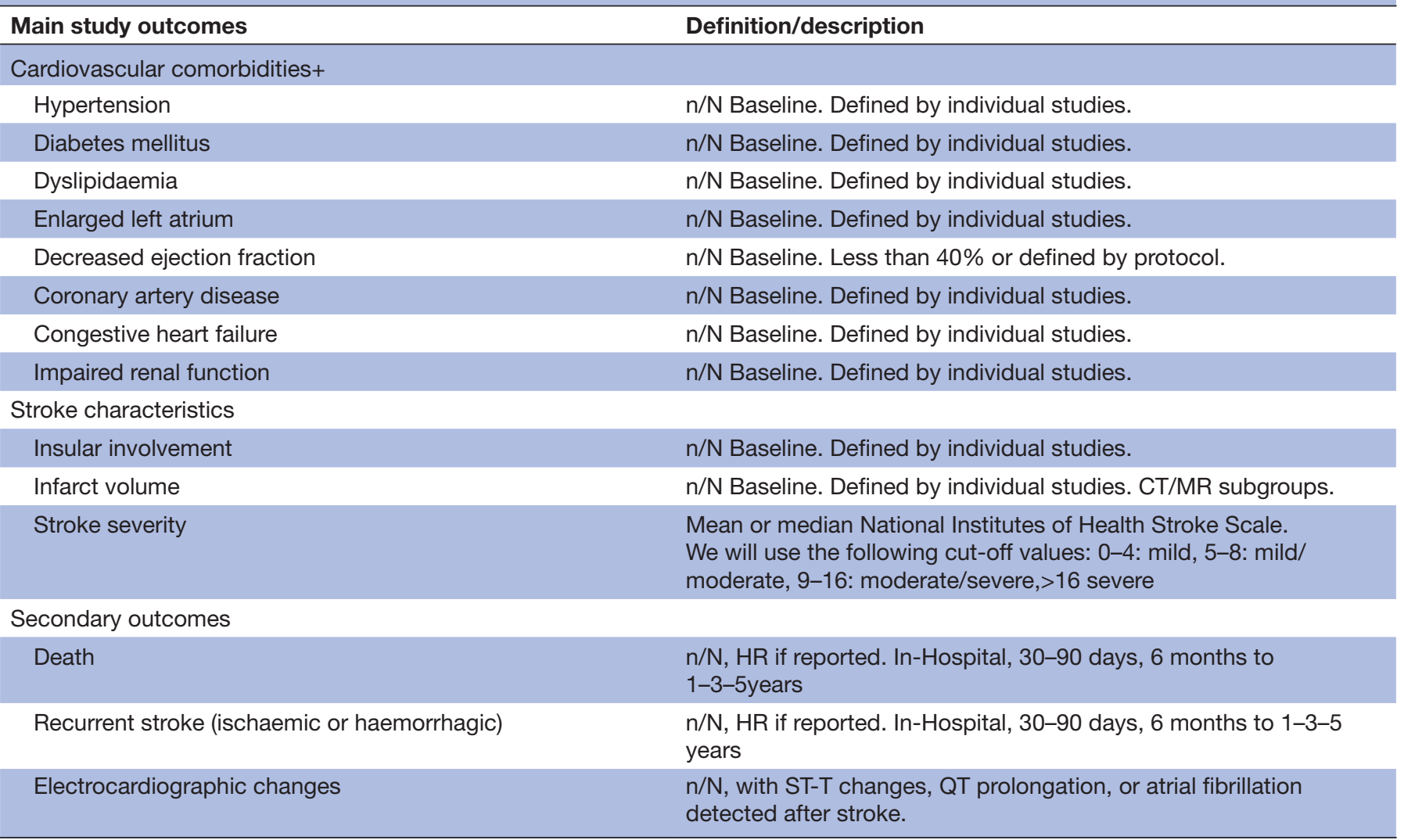

cTn-I vs cTn-T, standard versus high-sensitivity assay, cut-off value, mean or median interval time to cTn measurement.

- Study outcomes: study outcomes are described in table 4 .

\section{Data analysis and reporting}

We will normalise troponin levels to the respective 99th percentile in multiples of the 99th percentile when possible. We will apply random-effects or fixed-effects meta-analyses depending on the source of heterogeneity to estimate the proportion of IS and ICH patients with cTn elevation. For the main and secondary study objectives, we will report risk ratios when possible. Otherwise, we will report ORs. We will use the Agresti-Cuoll method to calculate confidence intervals for individual studies. We will calculate between study variance $\tau^{2}$ with the maximum-likelihood estimator and adjusted with the Hartung and Knapp method for calculations of between studies confidence intervals and adjusting test statistics. We will perform sensitivity analyses for subtypes of $\mathrm{cTn}$ (cTn-I and cTn-T), regular versus high-sensitivity assays, and type of stroke (IS vs ICH).

We will assess clinical heterogeneity by considering the unevenness in participants and study factors (prospective/retrospective, follow-up, cTn assay type). We will estimate heterogeneity across by using $\mathrm{t}^{2} Q$ and $\mathrm{I}^{2}$ measures. We will attempt to elucidate the basis of the heterogeneity by performing subgroup analysis. We will use the 'leave one out' procedure as a sensitivity analysis to identify studies responsible for heterogeneity. ${ }^{9}$ We will perform a combinatorial meta-analysis and we will apply a graphical display of study heterogeneity (GOSH) ${ }^{10}$ If outliers are found, we will enhance the GOSH plot with colour-code subgroup meta-analysis with and without the outlier study.

We will perform a meta-regression using the randomeffects model if data allows for exploring the different continuous variables.

We will evaluate whether selective reporting of outcomes is present. We will compare the fixed effect estimate against the random effects model to assess the possible presence of small sample bias in the published literature. We will apply enhanced funnel plots, Rosenthal's FailSafe N, Duval and Tweedie's trim and fill procedure, and Egger's regression intercept for evaluating reporting bias if at least 10 studies are retrieved.

We will conduct all analyses with R V.3.6.2 ( $\mathrm{R}$ Core Team,2014), by using the 'Meta' and 'Metaphor' packages according to the Cochrane Handbook for systematic reviews.

\section{Patient and public involvement statement}

No patients were involved in this study.

\section{Potential amendments}

We do not anticipate any amendment to this review protocol. However, any necessary amendment will be documented and reported transparently. 


\section{ETHICS AND DISSEMINATION}

This systematic review and meta-analysis will be based on published data and does therefore not require specific ethical approval or consent for participation. Patients or the public were not involved in the design, or conduct, or reporting, or dissemination plans of our research. The results will be published in peer-reviewed journals and presented at scientific conferences.

\section{Author affiliations}

${ }^{1}$ Heart \& Brain Lab, Western University, London, Ontario, Canada

${ }^{2}$ Department of Clinical Neurological Sciences, London Health Sciences Centre, London, Ontario, Canada

${ }^{3}$ Department of Epidemiology and Biostatistics, Western University, London, Ontario, Canada

${ }^{4}$ Department of Anatomy and Cell Biology, Western University, London, Ontario,

Canada

${ }^{5}$ Robarts Research Institute, Western University, London, Ontario, Canada

${ }^{6}$ Lawson Health Research Institute, London, Ontario, Canada

Twitter Sebastian Fridman @friseb and Luciano A Sposato @SposatoL

Contributors LAS conceived the study. SF and LS drafted the manuscript. LAS, SF and AJ-R revised the manuscript. All authors approved the final version. LAS is the guarantor of the review.

Funding LAS is supported by the Kathleen and Dr Henry Barnett Research Chair in Stroke Research (Western University, London, Canada); the Edward and Alma Saraydar Neurosciences Fund (London Health Sciences Foundation, London, Canada); and the Opportunities Fund of the Academic Health Sciences Centre Alternative Funding Plan of the Academic Medical Organisation of Southwestern Ontario (AMOSO) (Ontario, Canada).

Disclaimer None of these funding sources had a role in the design of this study protocol.

Competing interests None declared.

Patient consent for publication Not required.

Provenance and peer review Not commissioned; externally peer reviewed.

Supplemental material This content has been supplied by the author(s). It has not been vetted by BMJ Publishing Group Limited (BMJ) and may not have been peer-reviewed. Any opinions or recommendations discussed are solely those of the author(s) and are not endorsed by BMJ. BMJ disclaims all liability and responsibility arising from any reliance placed on the content. Where the content includes any translated material, BMJ does not warrant the accuracy and reliability of the translations (including but not limited to local regulations, clinical guidelines, terminology, drug names and drug dosages), and is not responsible for any error and/or omissions arising from translation and adaptation or otherwise.

Open access This is an open access article distributed in accordance with the Creative Commons Attribution Non Commercial (CC BY-NC 4.0) license, which permits others to distribute, remix, adapt, build upon this work non-commercially, and license their derivative works on different terms, provided the original work is properly cited, appropriate credit is given, any changes made indicated, and the use is non-commercial. See: http://creativecommons.org/licenses/by-nc/4.0/.

\section{ORCID iDs}

Amado Jimenez-Ruiz http://orcid.org/0000-0002-0257-3107

Sebastian Fridman http://orcid.org/0000-0002-1165-040X

Luciano A Sposato http://orcid.org/0000-0001-6425-9343

\section{REFERENCES}

1 Powers WJ, Rabinstein AA, Ackerson T. Guidelines for the early management of patients with acute ischemic stroke. Stroke 2018;2018:e46-110.

2 Sposato LA, Fridman S, Whitehead SN, et al. Linking stroke-induced heart injury and neurogenic atrial fibrillation: a hypothesis to be proven. J Electrocardiol 2018;S0022-0736:430-2.

3 Scheitz JF, Nolte $\mathrm{CH}$, Doehner W, et al. Stroke-heart syndrome: clinical presentation and underlying mechanisms. Lancet Neurol 2018;17:1109-20.

4 Sposato LA, Hilz MJ, Aspberg S, et al. Post-Stroke cardiovascular complications and neurogenic cardiac injury: JACC state-of-the-art review. J Am Coll Cardiol 2020;76:2768-85.

5 Broersen LHA, Stengl H, Nolte CH, et al. Association Between HighSensitivity Cardiac Troponin and Risk of Stroke in 96702 Individuals: A Meta-Analysis. Stroke 2020;51:1085-93.

6 Scheitz JF, Nolte CH, Laufs U, et al. Application and interpretation of high-sensitivity cardiac troponin assays in patients with acute ischemic stroke. Stroke 2015;46:1132-40.

7 Moher D, Shamseer L, Clarke M, et al. Preferred reporting items for systematic review and meta-analysis protocols (PRISMA-P) 2015 statement. Syst Rev 2015;4:1.

8 Schünemann HJ, Cuello C, Akl EA, et al. Grade guidelines: 18. How ROBINS-I and other tools to assess risk of bias in nonrandomized studies should be used to rate the certainty of a body of evidence. $J$ Clin Epidemiol 2019;111:105-14.

9 Viechtbauer W, Cheung MW-L. Outlier and influence diagnostics for meta-analysis. Res Synth Methods 2010;1:112-25.

10 Olkin I, Dahabreh IJ, Trikalinos TA. GOSH - a graphical display of study heterogeneity. Res Synth Methods 2012;3:214-23. 